\title{
Maternal ethnicity and risk of neural tube defects: a population-based study
}

\author{
Joel G. Ray, Marian J. Vermeulen, Chris Meier, David E.C. Cole, Philip R. Wyatt
}

Abstract

Background: Maternal body mass and the presence of diabetes mellitus are probable risk factors for neural tube defects (NTDs). The association between maternal ethnicity and the risk of NTDs remains poorly understood, however.

Methods: We performed a retrospective population-based study and included all women in Ontario who underwent antenatal maternal screening (MSS) at 15 to 20 weeks' gestation between 1994 and late 2000. Self-declared maternal date of birth, ethnicity and weight and the presence of pregestational diabetes mellitus were recorded in a standardized fashion on the MSS requisition sheet. NTDs were detected antenatally by ultrasonography or fetal autopsy and postnatally by considering all live and stillborn affected infants beyond 20 weeks' gestation. The risk of open NTD was evaluated across the 5 broad ethnic groups used for MSS, with white ethnicity as the referent.

Results: Compared with white women ( $n=290$ 799), women of First Nations origin $(n=1551)$ were at increased associated risk of an NTD-affected pregnancy (adjusted odds ratio [OR] $5.2,95 \%$ confidence interval [Cl] 2.1-12.9). Women of other ethnic origins were not at increased associated risk compared with white women (women of Asian origin [ $n=75$ 590]: adjusted OR 0.9, 95\% Cl 0.6-1.3; black women [ $n=25$ 966]: adjusted OR 0.6, 95\% Cl 0.3-1.1; women of "other" ethnic origin [ $n=10$ 009]: adjusted OR 0.1, 95\% Cl 0.02-0.9).

Interpretation: The associated risk of NTD-affected pregnancies was higher among women of First Nations origin than among women of other ethnic origins. The mechanisms for this discrepancy should be explored.

CMAJ 2004;171(4):343-5

$\mathrm{T}$

he risk of neural tube defects (NTDs) can be reduced by periconceptional folic acid supplementation. ${ }^{1}$ In previous US studies, the NTD rate varied with ethnicity, ${ }^{2,3}$ but potential confounders, including maternal weight ${ }^{4}$ and the presence of diabetes mellitus, ${ }^{5}$ were not controlled for. Because no data exist on the relationship between ethnicity and the occurrence of NTDs in pregnancies in Canada, including pregnancies of women of First Nations origin, we initiated the following study.

\section{Methods}

We performed a retrospective population-based study and included all women in Ontario who underwent antenatal maternal serum screening (MSS) between 1994 and late 2000. Since 1993, standardized MSS has been accessible to all women at 15 to 20 weeks' gestation through any physician or midwife, with a mean rate of uptake of more than $70 \% .{ }^{6}$ Self-declared maternal date of birth, ethnicity and weight and the presence of pregestational diabetes mellitus are recorded in a standardized fashion on the MSS requisition sheet, which is completed at the time of screening.

Women with a positive MSS are referred for counselling at 1 of 17 genetics centres in Ontario. Each centre contributes follow-up data to the Ontario MSS Database, which is funded by the Ontario Ministry of Health and Long-term Care. Anomalies were detected antenatally by ultrasonography or fetal autopsy and postnatally by considering all live and stillborn affected infants after 20 weeks' gestation. ${ }^{6}$ The latter were identified by data linkage of the mother's Ontario health insurance number with her infant's number assigned during the delivery hospital admission through the Canadian Institute for Health Information Discharge Abstract Database.

The risk of open NTDs was evaluated across the 5 broad ethnic groups used for MSS, with white ethnicity as the referent. Crude and multivariate odds ratios (ORs), along with $95 \%$ confidence intervals (CIs), were derived using unconditional logistic regression analysis. We adjusted for maternal age (1-year increments), weight at the time of MSS (1-kg increments), ${ }^{4}$ presence of pregestational diabetes ${ }^{5}$ and year of screening, each of which may influence either the uptake of MSS or the risk of NTDs. All variables were included in the model a priori. Participant identifiers were removed from the data set before analysis.

Permission to conduct this study was obtained through a research protocol approved by the Ontario Ministry of Health and Long-term Care and by the Research Ethics Board of St. Michael's Hospital.

\section{Results}

The characteristics of all 403915 women who underwent MSS are given in Table 1. Women of First Nations origin were younger, weighed more and had at least 3 times the rate of self-declared diabetes mellitus compared with women of other origins. Of all 276 NTDs reported, 202 $(73.2 \%)$ were identified antenatally (Table 1$)$.

Compared with white women, the associated risk of NTD-affected pregnancies was highest among women of First Nations origin (adjusted OR 5.2, 95\% CI 2.1-12.9) (Table 2). The lower associated risk of NTD-affected pregnancies among Asian women was no longer apparent after adjusting for potential confounders (adjusted OR 0.9, 95\% CI 0.6-1.3). There was little difference in the risk of 
NTD-affected pregnancies between white women and black women (adjusted OR 0.6, 95\% CI 0.3-1.1). Women of "other" ethnicity appeared to be at lower risk of NTDs (adjusted OR 0.1, 95\% CI 0.02-0.91), and only 1 NTDaffected pregnancy occurred in this group.

\section{Interpretation}

We observed a higher associated risk of NTD-affected pregnancies among Ontario women of First Nations origin who underwent MSS, something not seen in other ethnic groups. Because of the low event rates, the OR observed herein can be approximated by a relative risk estimate. Accordingly, the adjusted risk of NTD-affected pregnancy appears to be about 5 times higher among women of First Nations origin than among white women.

A relatively small number of First Nations women underwent MSS over the 7-year period of this study, such that these data may not be applicable across all ethnogenetic groups of First Nations peoples. The broad ethnic categories were generated for use within the MSS program and were not intended to mirror known ethnic stratifications sometimes used by population geneticists. They cannot account for the diversity within each group, nor do they identify birth origin or other factors such as socioeconomic status. Accordingly, we do not believe that maternal ethnicity should be used to modify the pretest probability of having an NTD-affected pregnancy. Some study participants may have had unrecognized type 2 diabetes, a risk factor for NTDs, ${ }^{5}$ but adjusting for maternal weight likely controlled for at least some of the potential confounding effect. ${ }^{7}$ Finally, by considering both antenatally and postnatally detected NTDs, we missed probably only a few events, even for pregnancies that resulted in a termination.

We did not determine who in our study was taking folic acid supplements. Unfortunately, there is a paucity of data about ethnic differences in folic acid intake among Canadian women and about differences in their nutritional status, especially among women belonging to the First Nations. In a study conducted in the Eastern James Bay region, mean erythrocyte folate concentrations were significantly lower among Cree women who had previously experienced an NTD-affected pregnancy than among unaffected Cree or white control subjects. ${ }^{8}$ Moreover, maternal case subjects had a lower estimated daily intake of foods containing folic acid, an observation that has been made elsewhere. ${ }^{9}$

If the risk of NTD-affected pregnancies is truly higher among women of First Nations origin than among women of other origins, then the mechanisms for this discrepancy should be elucidated. This might entail determining the rates of smoking and pregnancy planning, including screening for prepregnancy diabetes in high-risk groups. Canadian initiatives, including the First Nations Chiefs' Health Committee Pre-Natal Nutrition Program (www.fnchc.ca/CPNP.html), should continue to emphasize the importance of planned conception and higher dietary and supplemental folic acid intake periconceptionally. Assurance is also needed that all flour supplied to Canadians is fortified with folic acid, in accordance with federal law. ${ }^{6}$

\section{This article has been peer reviewed.}

From the Department of Medicine, St. Michael's Hospital (Ray), the Institute for Clinical Evaluative Sciences, Sunnybrook and Women's College Health Sciences Centre (Vermeulen), the Departments of Laboratory Medicine and Pathobiology,

Table 2: Risk of pregnancy affected by open neural tube defects by ethnicity

\begin{tabular}{lrccc}
\hline & \multicolumn{4}{c}{ Neural tube defects* } \\
\cline { 2 - 5 } Ethnicity & $\begin{array}{c}\text { No. }(\text { rate } \\
\text { per 1000) }\end{array}$ & $\begin{array}{c}\text { Crude OR } \\
(95 \% \mathrm{Cl})\end{array}$ & $\begin{array}{c}\text { Adjusted OR† } \\
(95 \% \mathrm{Cl})\end{array}$ \\
\hline White & $221(0.8)$ & 1.0 & 1.0 \\
Asian & $38(0.5)$ & $0.6(0.5-0.9)$ & $0.9(0.6-1.3)$ \\
Black & $11(0.4)$ & $0.6(0.3-1.0)$ & $0.6(0.3-1.1)$ \\
Other & $1(0.1)$ & $0.1(0.02-0.9)$ & $0.1(0.02-0.9)$ \\
First Nations & $5(3.2)$ & $4.2(1.8-10.3)$ & $5.2(2.1-12.9)$ \\
\hline
\end{tabular}

Note: $\mathrm{OR}=$ odds ratio, $\mathrm{Cl}=$ confidence interval.

*Isolated anencephalus, spina bifida with hydrocephalus and isolated spina bifida (International Classification of Diseases, 9th revision, diagnostic codes 740.0,

741.0 and 741.9).

†Adjusted for maternal age (1-year increments), weight (1-kg increments), presence of pregestational diabetes mellitus and year of screening.

Table 1: Characteristics of women in Ontario who underwent antenatal maternal serum screening between 1994 and late 2000, by ethnicity

\begin{tabular}{|c|c|c|c|c|c|}
\hline \multirow{2}{*}{ Characteristic } & \multicolumn{5}{|c|}{ Ethnicity } \\
\hline & $\begin{array}{c}\text { White } \\
n=290799\end{array}$ & $\begin{array}{c}\text { Asian } \\
n=75590\end{array}$ & $\begin{array}{c}\text { Black } \\
n=25966\end{array}$ & $\begin{array}{c}\text { Other } \\
n=10009^{*}\end{array}$ & $\begin{array}{l}\text { First Nations } \\
\quad n=1551\end{array}$ \\
\hline Age, mean (SD), yr & $30.1 \quad(5.0)$ & 30.5 (4.8) & $29.2 \quad(5.7)$ & $29.8 \quad(5.5)$ & $26.5 \quad(6.3)$ \\
\hline Weight, mean (SD), kg & $68.9(14.4)$ & $58.1(10.3)$ & $70.4(15.3)$ & $64.5(13.7)$ & $74.9(14.2)$ \\
\hline $\begin{array}{l}\text { Prevalence of pregestational } \\
\text { diabetes mellitus, \% }\end{array}$ & 0.53 & 0.33 & 0.54 & 0.69 & 2.0 \\
\hline $\begin{array}{l}\% \text { (no.) of neural tube } \\
\text { defects detected antenatally }\end{array}$ & $74.2(164 / 221)$ & $65.8(25 / 38)$ & $63.6(7 / 11)$ & $100.0(1 / 1)$ & $100.0(5 / 5)$ \\
\hline
\end{tabular}

*Includes 1324 women of Hispanic ethnicity. 
Medicine and Paediatrics (Genetics), University of Toronto (Cole), the Ontario Maternal Serum Screening Database Department, Genetics Programme (Meier) and the Department of Genetics (Wyatt), North York General Hospital, Toronto, Ont.

Competing interests: None declared.

Contributors: Joel Ray contributed to the study conception and design, the data acquisition, analysis and interpretation, and the drafting of the article. Marian Vermeulen contributed to the data analysis and interpretation. Chris Meier contributed to the data acquisition and analysis. David Cole contributed to the study conception and design, and the data interpretation. Philip Wyatt contributed to the study conception and design, the data acquisition and interpretation, and the drafting of the article. All authors contributed to the revision of the article and gave final approval of the version to be published.

Acknowledgements: We thank both the Ontario provincial laboratories and genetics clinics for contributing data to the Ontario MSS Database and the women of Ontario for supporting the maternal serum screening program. The Spina Bifida and Hydrocephalus Association of Canada and the Physicians' Services Incorporated Foundation supported this study.

\section{References}

1. Lumley J, Watson L, Watson M, Bower C. Periconceptional supplementation with folate and/or multivitamins for preventing neural tube defects. Cochrane Database Syst Rev 2001;CD001056.
2. Greene WB, Terry RC, DeMasi RA, Herrington RT. Effect of race and gender on neurological level in myelomeningocele. Dev Med Child Neurol 1991;33:110-7.

3. Feuchtbaum LB, Currier RJ, Riggle S, Roberson M, Lorey FW, Cunningham GC. Neural tube defect prevalence in California (1990-94): eliciting patterns by type of defect and maternal race/ethnicity. Genet Test 1999;3:265-72.

4. Werler MM, Louik C, Shapiro S, Mitchell AA. Prepregnant weight in relation to risk of neural tube defects. FAMA 1996;275:1089-92.

5. McLeod L, Ray JG. Prevention and detection of diabetic embryopathy. Community Genet 2002;5:33-9.

6. Ray JG, Meier C, Vermeulen MJ, Boss S, Wyatt PR, Cole DEC. Association of neural tube defects and folic acid food fortification. Lancet 2002;360:2047-8.

7. Mohamed N, Dooley J. Gestational diabetes and subsequent development of NIDDM in aboriginal women of northwestern Ontario. Int 7 Circumpolar Health 1998;57(Suppl 1):355-8.

8. Arbour L, Christensen B, Delormier T, Platt R, Gilfix B, Forbes P, et al Spina bifida, folate metabolism, and dietary folate intake in a northern Canadian aboriginal population. Int 7 Circumpolar Health 2002;61(4):341-51.

9. Moffatt ME. Current status of nutritional deficiencies in Canadian aboriginal people. Can 7 Physiol Pharmacol 1995;73:754-8

Correspondence to: Joel G. Ray, Department of Medicine and Inner City Health Research Unit, St. Michael's Hospital, University of Toronto, 30 Bond St., Toronto ON M5B 1W8; rayj@smh.toronto.on.ca

\section{CMAJ's Editorial Fellowship}

The CMAJ Editorial Fellowship, launched in 1998, provides an exciting opportunity for physicians early in their training to discover the inner workings of a leading medical journal. Applications are invited from recent medical graduates and residents who are interested in obtaining a rich experience in medical writing, editing and publishing. The fellow participates in all aspects of journal production, ranging from deciding which manuscripts to publish and working with authors to soliciting commentaries and review articles. Fellows are also expected to write extensively and are encouraged to develop theme issues, series or other journal innovations.

The position is full time for one year and is based at CMAJ's offices in Ottawa. The salary is based on the equivalent residency remuneration in Ontario.

The next round of applications is for the 2005 fellowship, which begins July 1,2005 . The application deadline is December 15, 2004.

For more information, please contact Dr. John Hoey, Editor, at john.hoey@cma.ca.

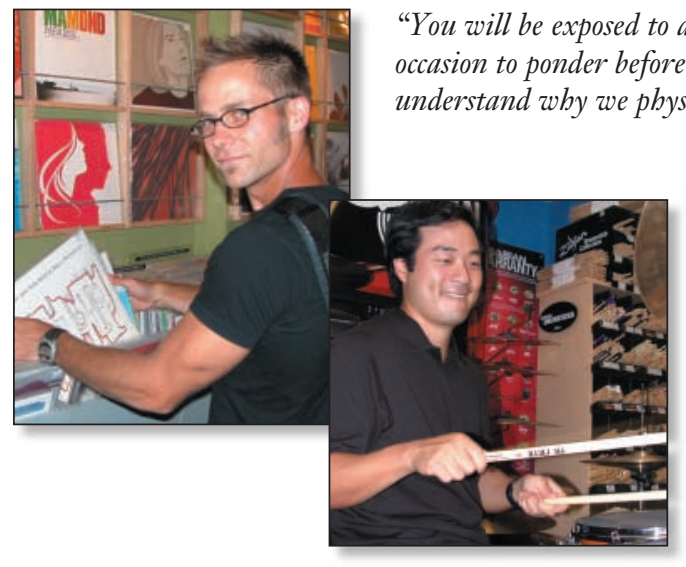

"You will be exposed to aspects of medicine that you never had occasion to ponder before. And, perhaps, you will better understand why we physicians do what we do."

— James Maskalyk, fellow 2002

"The fellowship is an amazing opportunity to develop new skills and learn what it means to be an editor. Prepare yourself for an exciting year as you watch new developments in modern medicine unfold before your eyes and help shape how physicians interpret them." 